\title{
DÉTECTION OPTIQUE DE I'A RÉSONANCE MAGNÉTIQUE NUCLÉAIRE DU MERCURE 201
}

\author{
Par B. CAGNAC, \\ Laboratoire de Physique, E. N. S., Paris.
}

\begin{abstract}
Résumé. - Les méthodes de pompage optique et de détection optique ont été employées précédemment pour orienter des atomes alcalins à l'état fondamental et pour étudier les résonances magnétiques entre sous-niveaux Zeeman et niveaux hyperfins de cet état.

Nous avons appliqué ces méthodes à l'orientation nucléaire de l'isotope $201 \mathrm{du}$ mercure dont l'état fondamental présente une configuration électronique diamagnétique (état ${ }^{1} S_{0}$ ). Cependant le noyau de cet isotope, de spin $I=3 / 2$, possède un moment magnétique dont l'orientation dans un champ extérieur présente quatre sous-niveaux Zeeman entre lesquels on peut induire des transitions de résonance magnétique nucléaire.

Nous obtenons l'alignement nucléaire dans la vapeur saturante pure (échantillon enrichi à $90 \%{ }^{201} \mathrm{Hg}$ ) en irradiant la vapeur parallèlement au champ magnétique avec la lumière non polarisée d'une source lumineuse émettant la raie $2537 \AA$ et contenant soit de l'isotope 198 (qui excite la composante hyperfine $3 / 2 \mathrm{de}^{201} \mathrm{Hg}$ ) soit de l'isotope 204 (qui excite la composante hyperfine $5 / 2$ de ${ }^{201} \mathrm{Hg}$ ). Sur une fréquence de résonance de 10 kilocycles par seconde, la résonance, détectée par changement de l'état de polarisation de"la lumière de résonance optique, se situe à $H_{0}=35,6$ gauss.

La mesure de la largeur des courbes de résonances (inférieure à 10 cycles par sec.) montre que les collisions entre les atomes de mercure et les parois de quartz de la cellule ne désorientent pas les noyaux alors que dans le cas des atomes alcalins, de configuration électronique paramagnétique (état ${ }^{2} S_{1 / 2}$ ) une telle désorientation a lieu.

Ceci constitue la première mesure directe du moment magnétique du noyau ${ }^{201} \mathrm{Hg}$.
\end{abstract}

Abstract. - Optical pumping and optical detection have been used extensively in the study of magnetic resonance of alkali metals (low and high frequency resonances).

We have applied these methods to produce nuclear alignment of ${ }^{201} \mathrm{Hg}$ in the $6{ }^{1} S_{0}$ ground state.)

Wed observed nuclear alignment in pure $\mathrm{Hg}$ vapour (enriched sample containing $90 \%$ of ${ }^{201} \mathrm{Hg}$ ) we illuminate it with impolarised light coming in the direction of the static magnetic field. The light source was filled with ${ }^{198} \mathrm{Hg}$ (we excite then only the $3 / 2$ component of ${ }^{201} \mathrm{Hg}$ ) or with ${ }^{204} \mathrm{Hg}$ (exciting the $5 / 2$ component only). At $10 \mathrm{Kc}$ nuclear magnetic resonance. is observed at about 35,6 gauss. The width of the observed lines (about 10 cycles) shows that collisions on the walls do not destroy orientation of mercury nuclei contrary to what is observed with alkali metals.

This is the first time nuclear magnetic resonance of ${ }^{201} \mathrm{Hg}$ has been observed.

La méthode de pompage optique [1] a été utilisée pour mettre en évidence la résonance magnétique dans l'état fondamental des atomes alcalins' $\mathrm{Na}, \mathrm{K}, \mathrm{Rb}, \mathrm{Cs}$ [2]. De nombreuses variantes du dispositif initial de pompage ont été proposées et mises en œuvre avec succès [3]. Rappelons que l'illumination d'une vapeur alcaline par sa raie de résonance polarisée circulairement provoque “l'orientation " de cette vapeur ; au contraire, l'illumination en lumière naturelle, dans la direction du champ magnétique, aboutit à un "alignement " partiel qui a pu être observé par Margerie [4].

Les atomes précédents sont paramagnétiques; en opérant sur un atome diamagnétique possédant un spin nucléaire, on peut espérer obtenir une orientation (ou un alignement) nucléaire en utilisant le même procédé. L'expérience, tentée par Brossel et Bitter [5] sur ${ }^{199} \mathrm{Hg}$, avait donné un résultat négatif. La raison de cet échec est sans doute le manque d'intensité de la lumière orientatrice : faible brillance de la source, manque d'ouverture des faisceaux utilisés, forte absorption

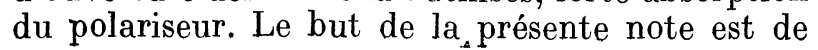

signaler que nous avons repris cette expérience sur ${ }^{201} \mathrm{Hg}$ de spin nucléaire $I=3 / 2$ et que nous avons obtenu un résultat positif.

L'isotope utilisé, séparé au spectrographe de masse à double monochromateur du C. E. A., par M. Cassignol, avait une concentration de $90 \%$; il était contenu dans une cellule cubique en quartz fondu de $4 \mathrm{~cm}$ d'arête ; la température du réservoir de mercure était $-20^{\circ} \mathrm{C}$ de manière à éviter les effets de la diffusion multiple.

L'excitation optique utilise la lumière naturelle, l'illumination se faisant dans la direction du champ magnétique (composantes $\sigma^{+}$et $\sigma^{-}$) de manière à obtenir un alignement partiel du moment nucléaire. La source est une lampe sans électrodes de $20 \mathrm{~mm}$ de diamètre et de $1 \mathrm{~mm}$ d'épaisseur ; elle a la forme d'un disque très plat et est observée perpendiculairement aux faces. Elle contient soit du mercure ${ }^{198} \mathrm{Hg}$ (à $98 \%$ ), soit du mercure ${ }^{204} \mathrm{Hg}$ (à $98 \%$ ) et est excitée à l'aide d'un magnétron fournissant 100 watts à $3000 \mathrm{mc} / \mathrm{s}$.

La structure de la raie $2537 \AA$ est telle que les composantes de ${ }^{198} \mathrm{Hg}$ et ${ }^{204} \mathrm{Hg}$ coincident à peu 
près avec les composantes hyperfines de ${ }^{201} \mathrm{Hg}$ conduisant respectivement aux états hyperfins $\mathrm{F}=3 / 2$ et $F=5 / 2$ du niveau excité $6^{3} P_{1}[6]$.

L'alignement de ${ }^{201} \mathrm{Hg}$ est donc produit par

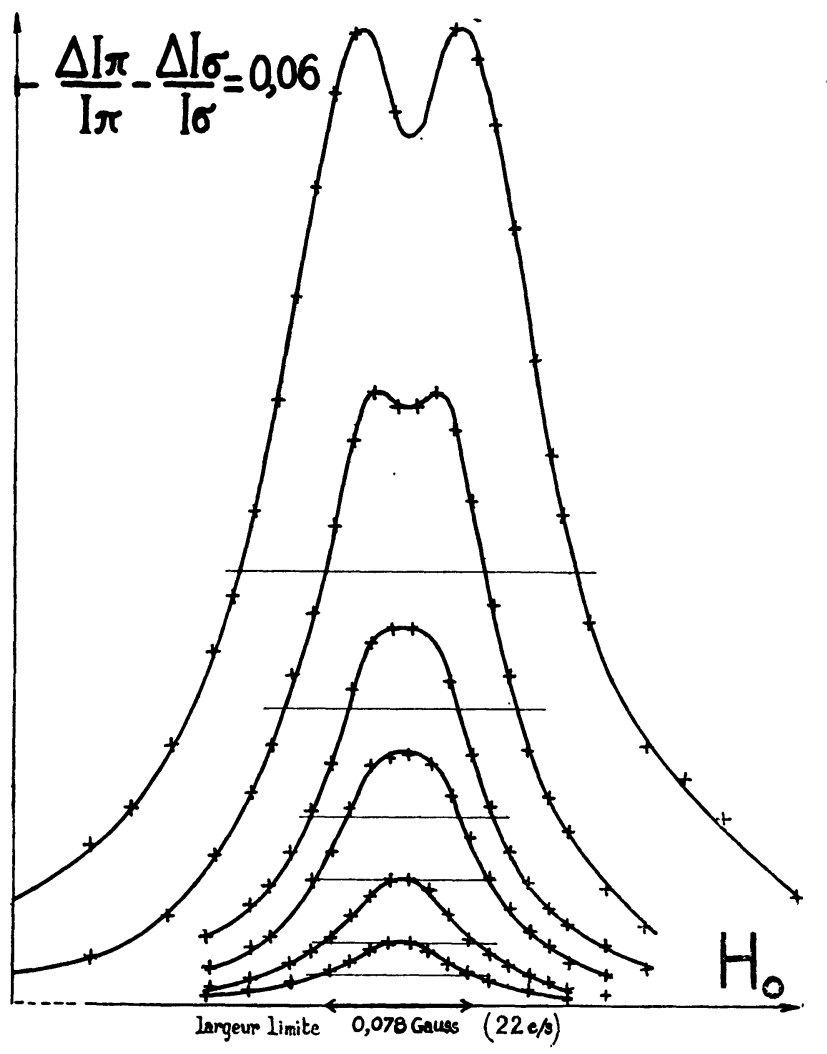

Fig. 1.

absorption d'une seule composante $3 / 2$ ou $5 / 2$ et les valeurs des probabilités de transition optiques dans les deux cas sont telles que les niveaux $m_{\mathrm{I}}= \pm 1 / 2$ de l'état fondamental se vident partiellement au profit des niveaux $m_{\mathbf{I}}= \pm 3 / 2$. La détection de la résonance magnétique entre

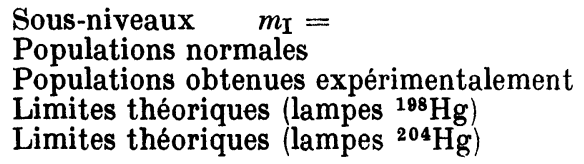

La largeur $\Delta v$ des raies observées est très faible. Elle dépend du niveau de radiofréquence $H_{1}$, de la "durée de vie " $T$ des niveaux $m_{\mathrm{I}}$ de l'état fondamental et des inhomogénéités $\Delta H_{0}$ du champ $H_{0}$ sur le volume de la cellule.

La mesure de la largeur $\Delta v$ en fonction de $H_{1}$ permet d'extrapoler la largeur $\Delta v_{0}$ pour $H_{1}$ nul. $\Delta v_{0}$ est de 22 cycles par seconde pour les courbes de la figure 1. Cela correspond à une "durée de vie " $T=1 / \pi \Delta v_{0}$ qui est environ 100 fois plus longue que le temps de transit d'un atome d'une paroi à niveaux $m_{\mathbf{I}}$ se fait par procédé optique : elle utilise le changement dı degré de polarisation de la. lumière de résonance optique réémise par la vapeur de ${ }^{201} \mathrm{Hg}$ (en l'absence de tout gaz étranger) sous l'influence d'un champ de radiofréquence. $H_{1}$, perpendiculaire au champ statique $H_{0}$.

Deux photomultiplicateurs mesurent respectivement les intensités $\mathrm{I}_{\pi}$ et $I_{\sigma}$ en polarisations $\pi$ et $\sigma$ de la lumière de résonance optique. Et leur montage en opposition dans un pont suivant le procédé classique [8] permet la mesure directe au galvanomètre d'un signal proportionnel à la différence des variations relatives de $I_{\pi}$ et $I_{\sigma}$ provoquée par le champ de radiofréquence : $\frac{\Delta I_{\pi}}{I_{\pi}}-\frac{\Delta I_{\sigma}}{I_{\sigma}}$. C'est cette quantité qui est portée en ordonnée sur la figure 1 représentant les résonances observées avec la lampe de ${ }^{198} \mathrm{Hg}$ pour un champ oscillant $H_{1}$ de fréquence $10 \mathrm{kHz}$ et de diverses amplitudes. Les courbes sont bien représentées par les formules de Majorana-Brossel [8] et présentent aux fortes valeurs de $H_{1}$ le renversement typique.

Nous avons utilisé les fréquences de $5,10,25$ et $100 \mathrm{KHz}$. La résonance a été observée pour les valeurs respectives du champ statique $H_{0}$ de 17,8 ; 35,$6 ; 89,0$; et 356 gauss (connues à $0,5 \%$ près) aux emplacements prévus.

Les variations $\Delta I_{\pi}$ et $\Delta I_{\sigma}$ à la résonance ont le signe prévu : $\Delta I_{\pi}$ est toujours positif; tandis que $\Delta I_{\sigma}$ est positif ou négatif suivant que l'on utilise respectivement la lampe ${ }^{198} \mathrm{Hg}$ ou la lampe ${ }^{204} \mathrm{Hg}$. Le signal différence $\frac{\Delta I_{\pi}}{I_{\pi}}-\frac{\Delta I_{\mathbf{\sigma}}}{I_{\sigma}}$ est toujours positif.

La grandeur du signal à saturation, pour une grande amplitude de $H_{1}$ permet d'évaluer les changements de populations des sous-niveaux fondamentaux, obtenus par le pompage optique. L'utilisation des lampes ${ }^{204} \mathrm{Hg}$ et ${ }^{198} \mathrm{Hg}$ nous a conduit à des évaluations voisines et que nous confondons dans le tableau ci-dessous :

$\begin{array}{cccc}-3 / 2 & -1 / 2 & +1 / 2 & +3 / 2 \\ 1 & 1 & 1 & 1 \\ 1,15 & 0,85 & 0,85 & 1,15 \\ 1,30 & 0,70 & 0,70 & 1,30 \\ 1,50 & 0,50 & 0,50 & 1,50\end{array}$

l'autre : les collisions contre les parois sont donc sans influence sur le spin nucléaire. Elles ne sont pas une cause importante de désorientation contrairement à ce qui se produit pour les valeurs alcalines.

Aux diverses fréquences les largeurs extrapolées $\Delta v_{0}$ sont les mêmes dans les limites de prëcision auxquelles nous pouvons prétendre (environ $10 \%$ ). L'inhomogénéité $\Delta H_{0}$ du champ directeur $H_{0}$ n'entre donc que pour une part négligeable dans les largeurs des résonances.

Le temps $T$ est en grande partie déterminé par 
l'intensité lumineuse $I$, comme le montre la figure 2. On voit que $1 / T$ est une fonction linéaire de $I$ comme on doit s'y attendre : $T$ est en effet au plus

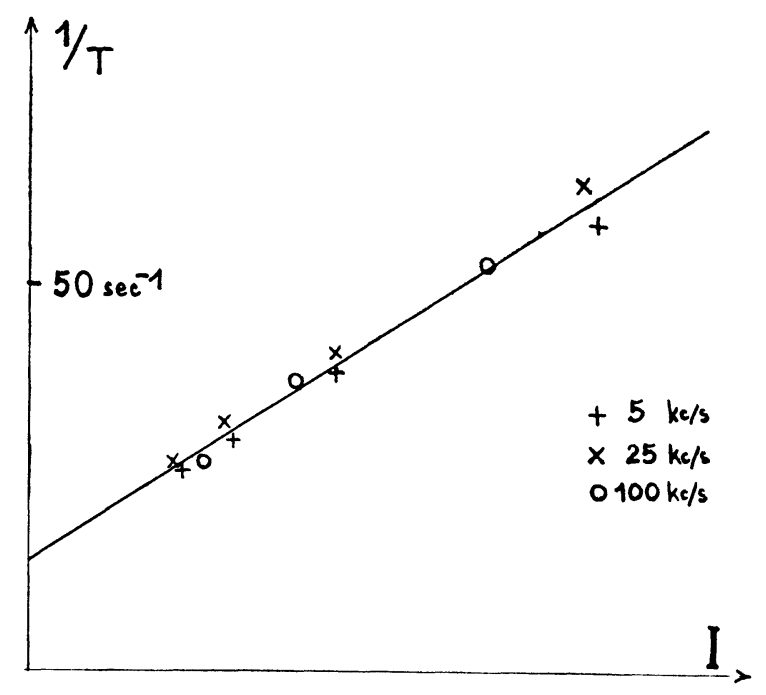

Fig. 2. - En ordonnées, l'origine correspond à $\frac{1}{T}=15 \mathrm{~s}^{-1}$.

égal au temps moyen qui sépare l'absorption successive de 2 photons par le même atome. Aux intensités lumineuses très faibles, $\Delta \nu_{0}$ est de l'ordre de $8 \mathrm{c} / \mathrm{s}$ ce qui correspond à une "durée de vie " $T=0,04$ seconde. Nous n'avons pas encore identifié avec certitude l'origine de la relaxation.

\section{BIBLIOGRAPHIE}

[1] Kastler (A.), J. Physique Rad., 1950, 11, 255. Andrew (E. R.), Nuclear Magnetic Resonance, Cambridge Un. Press, 1955, 63.

[2] Kastler (A.), J. Opt. Soc. Amer., 1957, 47, 460 et Nuovo Cimento, 1957, 6, no 3 del Supplemento, 1148. Arditi (M.) et Carver (T. R.), Phys. Rev., 1958, 109, 1012. Skalinski (T. S.), C. R. Acad. Sc., 1957, 245, 1908. Diamand (F.), Legendre (J. M.) et Skalinski (T.), C. R. Acad. Sc., 1958, 246, 90. Hartmann (F.), Rambosson (M.), Brossel (J.) et Kastler (A.), C. R. Acad. Sc., 1958, 246, 1522.

[3] Brossel (J.), Margerie (J.) et Kastler (A.), $C . R$. Acad. Sc:, 1955, 241, 865. Dehmelt (H. G.), Phys. Rev., 1957, 105, 1487. Bell (W. E.) et Bloom (A. L.), Phys. Rev., 1957, 107, 1559 et 1958, 109, 219. FranZen (W.) et Emslie (A. G.), Phys. Rev., 1957, 108, 1453.

[4] Margerie (J.), Brossel (J.) et Kastler (A.), C. R. Acad. Sc., 1955, 241, 474.

[5] Bitter (F.) et Brossel (J.), Phys. Rev., 1952, 85, 1051. Bitter (F.), Lacey (R. F.) et Richter (B.), Rev. Mod. Physis, 1953, 25, 174.

[6] Sagalyn (P. L.), Melissinos (A. G.) et Bitter (F.), Phys. Rev., 1958, 109, 375.

[7] Proctor (W. G.) et Yu (F. C.), Phys. Rev., 1951, 81, 20. Blaise (J.) et Chantrel (H.), J. Physique Rad., 1957, 18, 193.

[8] Brossel (J:) et Bitter (F.), Phys. Rev., 1952, 86, 308. Brossel (J.), Ann. Physique, 1952, 7, 622. 